\title{
Mathematical Modelling for Electromagnetic Field and Shaping of Melts in Cold Crucible
}

\author{
Tsutomu TANAKA, Kohichi KURITA and Atsuhiko KURODA1)
}

Iron and Steel Research Laboratories, Sumitomo Metal Industries, Ltd., Sunayama, Hasakimachi, Kashima-gun, Ibaraki-ken, 314-02 Japan. 1) Advanced Technology Research Laboratories, Sumitomo Metal Industries, Ltd., Fuso-cho, Amagasaki, Hyogo-ken, 660 Japan.

(Received on August 9, 1990; accepted in the final form on November 16, 1990)

\begin{abstract}
For the better understanding of the phenomena in the cold crucible, advanced models for electromagnetic field have been developed for both vertical and horizontal cross sections of the crucible. In the former model, the axi-symmetric sheet geometry was assumed and the influence of the number of slits was taken into account by the use of a parameter representing the magnitude of the electromagnetic interaction. In the latter one, the conservation problem of the current density in a finite cell was successfully solved through the introduction of Newton's method for the simultaneous convergence of both scalar and vector potentials. The validity of the models was confirmed through experimental measurements of the magnetic flux density, the coil voltage, and the elevation height of the $\mathrm{Al}-\mathrm{Cu}$ alloy melts.

Regardless of the number of slits and the shape of the crucible, the induced current on the internal and the external sheets of the crucible flows on the horizontal path. An increase in the number of slits resulted in the increase of the magnetic flux density. This is mainly explained by the reason that the partition ratio of the induced current into the segmented part of the crucible increases with the number of slits. Since the melt is expected to contact with the internal wall near the slit bottom in the case of the straight type crucible, the solidification is preferable to be completed just above the slit bottom for the improvement of the ingot surface during continuous casting.
\end{abstract}

KEY WORDS: cold crucible; electromagnetism; liquid metal; shaping; mathematical model.

\section{Introduction}

Molten metal in the cold crucible ${ }^{1,2)}$ is prevented from contacting with the wall of the crucible with the aid of pinch force exerted on the melt. The pinch force is generated by the induced current on a number of vertically arranged water cooled segments with slits surrounded by a multi-turn coil. Stirring and super heating of the melt are also promoted in the crucible. Hence, the cold crucible technique offers many varieties of application such as casting of chemically active materials with high melting temperature, waste treatment of radio active materials and production of small particles by the use of rapid quench of melt vapour.

Delage et al. ${ }^{3,4)}$ studied electromagnetic and thermal characteristics of cylindrically straight crucible. The approach to the problem was global and led to the determination of the resistance and the self inductance equivalent to the crucible. Although it is useful for designing a continuous casting system of the crucible, it cannot give a precise description for the other situations such as axi-symmetrically convergent crucibles in which the magnetic field gradient cannot be neglected.

Gagnoud et al ${ }^{5,6)}$ theoretically studied the magnetic flux density and the shape of the melt in the cold crucible by using the distributed system. However, the effects of the number of slits and the finite size of the coil on the electromagnetic field were not con- sidered and the coil voltage could not be determined in the model.

Two kinds of approaches to the mathematical modelling can be considered which allow the description of the shape of the melt with the melt flow as well as the heat transfer in an axi-asymmetric geometry of the crucible. The first approach to the modelling is based on the volume element model with the axiasymmetric geometry, while the second one is based on the simplified sheet element model with the axisymmetric geometry with modification for the presence of the slits. In the former model, however, it would arise difficulties especially in the determination of the shape of the melt with the temporal computer.

The aim of the present study is to develop the advanced mathematical model which enables to describe the relation among 1) the important parameters of the crucible, such as the shape of the crucible, number of slits and the volume of the melts, 2) the magnetic flux density, 3) the shape of the melts, and 4) the coil voltage. Another aim of this study is to clarify the path of the induced current, contact characteristic between the melt and the crucible and the anisotropy of the magnetic flux density due to the presence of slits.

\section{Electromagnetic Modelling}

\subsection{Model on the Vertical Section of the Crucible}

Basic idea in the development of the crucible model 
on the vertical section, which is especially effective in the determination of the melt shape, is as follows.

(1) Electromagnetic variables are defined on the surface of the conductor on the assumption of the axisymmetric sheet geometry with only one piece of slit whose thickness is infinitesimally small.

(2) The effect of the presence of a number of slits on the magnetic field is considered through the introduction of parameters which modifies the axi-symmetric sheet model.

\subsubsection{Governing Equations of Vector Potential}

Lorentz gauge should be applied on the relation between the vector potential and the scalar potential in the analysis of variables which are defined on the conductor surface and are used for the description of the internal structure. If vector potential is written as a monochromatic wave, $\mathbf{A}=\mathbf{A}_{0} e^{-i \omega t}$, the equation for the Green's function, which tends to zero when $|\mathbf{r}-\mathbf{R}|$ becomes infinite, is written as Eq. (1). ${ }^{7)}$

$$
G_{0}(\mathbf{r}, \mathbf{R})=\frac{1}{4 \pi|\mathbf{r}-\mathbf{R}|} \exp \{(i-1)|\mathbf{r}-\mathbf{R}| / \delta\}
$$

Because the crucible is assumed to be axi-symmetric, the Green's function $G_{0}$ can be integrated over the plane normal to the surface shown in Fig. 1. Since the condition $\delta \ll\left|\mathbf{R}_{1}-\mathbf{R}_{0}\right|$ usually holds in the operational frequency range of the crucible, a denominator is not the predominant term and is approximately written as $4 \pi\left|\mathbf{r}-\mathbf{R}_{0}\right|$ in the integration normal to the conductor surface $\mathbf{n}$. When the integration along the azimuthal direction is conducted, the generality is not lost because of the axial symmetry even if the observation point is restricted in the $x-z$ plane. Only the non-vanishing variable is its $\theta \mathrm{com}$ ponent. Hence,

$$
\begin{aligned}
G(x, z, X, Z) & =\int_{R_{1}}^{R_{0}} \int_{0}^{2 \pi} G_{0}(\mathbf{r}, \mathbf{R}) d \mathbf{n} d l_{y} \\
& =\frac{(1+i) \delta}{8 \pi} \int_{0}^{2 \pi} \frac{R_{0} \cos 0 d \theta}{\left|\mathbf{r}-\mathbf{R}_{0}\right|}
\end{aligned}
$$

where, the last term in the equation is obtained from the condition $\delta \ll\left|\mathbf{R}_{1}-\mathbf{R}_{0}\right|$. The integration in the

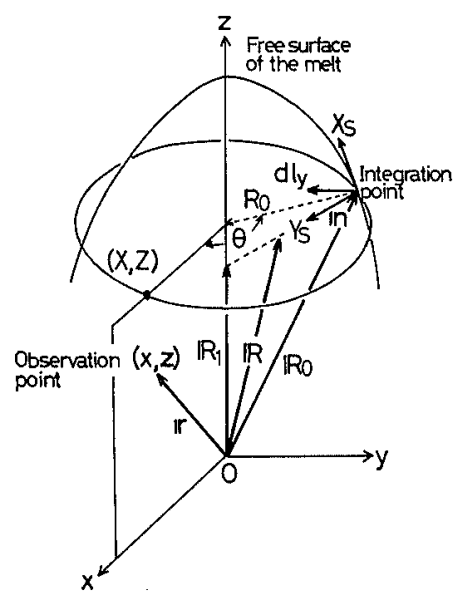

Fig. 1. Coordinate system in the integration of Green's function. last term of Eq. (2) is represented by the use of an elliptic function. ${ }^{8)}$

Vector potential is written as the integral form by introducing the Green's function,

$$
A(x, z)=\mu \int G(x, z, X, Z) j(X, Z) d l(X, Z)
$$

where, suffix 0 is eliminated from the variables for brevity.

Since the relation between the vector potential and the current density is written as Eq. (4),

$$
j(x, z)=-\sigma\left\{\frac{1}{x} \frac{\partial \phi}{\partial \theta}-i \omega A(x, z)\right\}
$$

Eqs. (3) and (4) constitute the integral equations with the unknown variables of the vector potential and the current density, when $1 / x \cdot \partial \phi / \partial \theta$ is given. In the present method, the coil current is excited by the homogeneous scalar potential applied on the coil edge without the discrimination of the intrinsic and the induced currents, which is different from the previous study. ${ }^{9}$ This makes it possible to calculate the vector potential and the current density when the current distribution in the finite size of the coil has to be considered. Scalar potential of the coil is given as the boundary condition and that of the crucible is determined from the conservation law of the current.

\subsubsection{Parameters in the Model of Vertical Section}

There is a thought that the current flows on the shortest path in a closed surface composed of the internal and the external sheets of the crucible. Especially, the path is horizontal when the number of slits is large. On the other hand, there is another thought that the reduction of the current density due to the presence of scalar potential between the adjacent slits should be taken into account when the current on the shortest path increases.

The model based on the former thought is called as the model 1, while that based on the latter is called as the model 2. A model between the model 1 and the model 2 is called as the intermediate model. The governing equations for the current density and the scalar potential on the external and the internal sheets are written as follows.

(1) Model 1

$$
\left.\begin{array}{l}
j_{1}^{\prime}\left(x_{1}, z_{1}\right)+j_{2}^{\prime}\left(x_{2}, z_{2}\right)=0 \\
\Delta \phi_{1}^{\prime}\left(x_{1}, z_{1}\right)=\Delta \psi_{2}^{\prime}\left(x_{2}, z_{2}\right)=0
\end{array}\right\}
$$

Here, suffixes 1 and 2 represent the internal and the external sheets, respectively.

(2) Model 2

$$
\left.\begin{array}{l}
\int j_{1}^{\prime \prime}\left(x_{1}, z_{1}\right) d l+\int j_{2}^{\prime \prime}\left(x_{2}, z_{2}\right) d l=0 \\
\Delta \phi_{1}^{\prime \prime}\left(x_{1}, z_{1}\right)=\Delta \phi_{2}^{\prime \prime}\left(x_{2}, z_{2}\right) \neq 0
\end{array}\right\}
$$

Here, $\Delta \phi^{\prime \prime}$ is obtained from the conservation law of the current. 
(3) Intermediate Model

$$
\left.\begin{array}{l}
j_{1}\left(x_{1}, z_{1}\right)=j_{1}^{\prime \prime}\left(x_{1}, z_{1}\right)\left(1-P_{12}\right)-j_{1}^{\prime}\left(x_{1}, z_{1}\right) P_{12} \\
\Delta \phi_{1}\left(x_{1}, z_{1}\right)=\Delta \phi_{1}^{\prime \prime}\left(x_{1}, z_{1}\right)\left(1-P_{12}\right)
\end{array}\right\}
$$

Here, $P_{12}$ is the first parameter which represents the degree of dependency of the current in the internal sheet on that in the external one and takes its value from 0 to 1 . The similar equation is obtained for $j_{2}$.

The second parameter $P_{i}$ is introduced to express the intensity of the electromagnetic interaction on the slit part of the crucible. Accordingly, the Green's function in Eq. (2) is rewritten as Eq. (8),

$$
G(x, z, X, Z) \rightarrow P(x, z) P(X, Z) G(x, z, X, Z)
$$

where, $P(x, y)=P_{i}$ when the coordinate $(x, y)$ corresponds to the slit and $P(x, y)=1$ in the other case.

\subsubsection{Shape of the Melt}

Shape of the melt is obtained by applying the Line Segments method ${ }^{10)}$ together with the local rotation of the coordinate to the sheet coordinate on the assumption that the metal is completely melted. The Local method ${ }^{6}$ is used to determine the shift direction of the coordinate, in which the equilibrium of forces exerted each grid is considered. The amount of the coordinate shift $\Delta Y_{s}$ normal to the surface is assumed to be determined from the static equilibrium condition of the electromagnetic force, surface tension and gravitational force, that is,

$$
\Delta Y_{S} \propto P_{M}+P_{S}+P_{I I}+P_{0}
$$

Here,

$$
\begin{aligned}
& P_{M}=\frac{\mu}{4} \delta^{2} \operatorname{Re}\left(j \cdot j^{*}\right), \\
& \left.\begin{array}{rl}
P_{S} & =\alpha\left\{\frac{d^{2} Y_{s} / d X_{s}^{2}}{\left[1+\left(d Y_{s} / d X_{s}\right)^{2}\right]^{3 / 2}}+\frac{1}{X \sqrt{1+1 /\left(d Y_{s} / d X_{s}\right)^{2}}}\right\}, \\
P_{H} & =\rho g\left(Z-Z_{0}\right), g<0,
\end{array}\right\}
\end{aligned}
$$

$P_{0}$ : the uniform pressure determined from the conservation condition of the melt volume.

\subsubsection{Calculation Method}

The iterative calculation for the current density was performed after eliminating the vector potential from Eqs. (3) and (4). The main boundary conditions are 1) conservation of the melt volume, 2) homogeneous scalar potential on the coil edge, 3 ) conservation of the current between the internal and the external sheets of the crucible, and 4) sum of the electromagnetic force, gravitational force and surface tension are uniform over the grids representing the free surface. Main calculated items are the current density, the scalar potential in the conductor and the coordinate

\begin{tabular}{|c|c|c|}
\hline & $\mathrm{Al}-\mathrm{Cu}$ alloy & Ti-Al alloy \\
\hline Melting temperature $\left({ }^{\circ} \mathrm{C}\right)$ & 650 & 1650 \\
\hline Density $\quad\left(\mathrm{kg} / \mathrm{m}^{3}\right)$ & 2570 & 4500 \\
\hline Electric conductivity (S/m) & $4.05 \times 10^{6}$ & $6.88 \times 10^{5}$ \\
\hline Surface tension $\quad(\mathrm{N} / \mathrm{m})$ & 0.84 & 1.51 \\
\hline
\end{tabular}
of the melt surface. Physical properties for Al-Cu and $\mathrm{Ti}-\mathrm{Al}$ alloys used in the calculation are shown in Table 1 .
Table 1. Physical properties of liquid alloys.

\subsection{Horizontal Model of the Cold Crucible}

2.2.1. Groverning Equations for Vector and Scalar Potentials

In the horizontal model, electromagnetic analysis is performed in the cell arranged inside of the conductor on the assumption that the cylindrical crucible is infinitely long surrounded by a single turn coil. The shape of the melt is not obtained in this model. Coulomb gauge should be applied to the Maxwell's equations for the cell which has no internal structure. The integral form for the equation of the vector potential in the case of line source is written as Eq. (11),

$$
\mathbf{A}(\mathbf{r})=-\frac{\mu}{2 \pi} \int_{0}^{2 \pi} \mathbf{j}(\mathbf{R}) \ln |\mathbf{r}-\mathbf{R}| \cos \theta d \mathbf{R}
$$

where, $\theta$ is the angle between vectors $\mathbf{A}$ and $\mathbf{j}$.

Since the relation between $\mathbf{A}$ and $\mathbf{j}$ is expressed as Eq. (12),

$$
\mathbf{j}=-\sigma\left\{\operatorname{grad}\left(\phi+\phi^{\oplus \times \mathrm{x}}\right)-i \omega \mathbf{A}\right\}
$$

$\mathbf{A}$ and $\mathbf{j}$ are obtained in combination with the Eq. (11) when $\phi$ is known and $\phi^{\mathrm{ext}}$ is given as a boundary condition.

The governing equation of the scalar potential is closely related with the conservation law of the current density. One of the approaches to obtain the scalar potential is based on the equation derived by the elimination of the current density from Eq. (12).

$$
\nabla^{2} \phi=i \omega \operatorname{div} \mathbf{A}-\nabla^{2} \phi^{\mathrm{ext}}
$$

Eq. (13) together with Eqs. (11) and (12) constitutes the mathematical description which determines the scalar potential as well as the current density and the vector potential. However, it should be noted that Eq. (13) is not so effective in the calculation because the scalar potential is not simultaneously obtained with the vector potential.

In the case of using Eq. (13), the scalar potential is obtained after the calculation of the vector potential. This means that the scalar and the vector potentials do not precisely satisfy the conservation law of the current density.

Problem associated with the simultaneous evaluation of the scalar and the vector potentials in electromagnetism is analogous to that of the simultaneous evaluation of the pressure and the velocity vector in fluid dynamics ${ }^{11)}$ except for being the complex variables in the case of the electromagnetism. The complex variable $\varepsilon$ is defined by Eq. (14) as,

$$
\varepsilon=\operatorname{div} \mathbf{j}=f(\phi)
$$

where $f$ is a function which almost depends on the 
scalar potential because the Green's function becomes predominantly diagonal in many cases. Correction of the scalar potential is evaluated from Newton's method as Eq. (15).

$$
\Delta \phi=-\varepsilon / \operatorname{det}\left(\frac{\partial \varepsilon}{\partial \phi}\right)
$$

Since $\varepsilon$ and $\phi$ are complex variables, $\partial \varepsilon / \partial \phi$ becomes Jacobian, which is different from the analysis in fluid dynamics. Correction of the current density is written as follows.

$$
\Delta \mathbf{j}=\frac{\partial \mathbf{j}(\phi)}{\partial \phi} \Delta \phi
$$

\subsubsection{Calculation Procedure}

(1) The scalar potential is allocated on the centre of the cell, while the vector potential and the current density are allocated on the cell boundary.

(2) In the first step, temporary current density is obtained from Eqs. (11) and (12).

(3) Secondary, the residual $\varepsilon$ is evaluated from Eq. (14) until it becomes less than an admissible value, followed by the consecutive correction of variables from Eqs. (15) and (16).

(4) Processes (2) and (3) are repeated until the residuals for the current density and for the scalar potential become less than admissible values.

\section{Experimental Method}

A system of the cold crucible consists of a five-turn water cooled copper coil with $10 \mathrm{~mm}$ in diameter and a water cooled crucible made of copper with $45 \mathrm{~mm}$ in internal diameter, $65 \mathrm{~mm}$ in external diameter, and $150 \mathrm{~mm}$ in height. A cylindrically straight crucible has periodically arranged 16 pieces of vertical slits along the circumferential direction in the middle height of the crucible. Width and length of the slits are 1 and $55 \mathrm{~mm}$, respectively. Most of the experiments were performed with the straight type crucible. Convergent type crucibles whose internal diameter at the slit top was $45 \mathrm{~mm}$ and that at the slit bottom was $30 \mathrm{~mm}$ or $8 \mathrm{~mm}$ were also used in combination with the crucible with 1,8 , or 16 slits.

The maximum output of the high frequency generator used is $65 \mathrm{~kW}$ and the frequency is $20 \mathrm{kHz}$. The frequency was measured by the use of an oscilloscope. The inductance was evaluated from the resonance condition and the coil current was theoretically obtained from the reactance and the applied voltage.

Main items of measurements are follows.

\section{(1) Magnetic Flux Density}

A search coil was used to measure the magnetic flux density. The device is a 10-turn coil of copper wire with $0.05 \mathrm{~mm}$ in diameter insulated by polyfluoroethylene and is wound around a rod of $5 \mathrm{~mm}$ in diameter. To minimize the error during measurements, leading wire part of the search coil was twisted.

(2) Elevation Height of Melt

Fixed amount of Al-Cu alloy was melted on the refractory placed below the slit bottom for the easiness of melting in the atmosphere of Ar gas using the crucible with 16 pieces of slits. After being melted, an aluminum plate of $0.5 \mathrm{~mm}$ thickness was inserted into the crucible to obtain the elevation height of the melt by the remained shape of the plate.

\section{(3) Energy Balance}

A Ti-Al alloy with $44 \mathrm{~mm}$ in diameter and 200 $\mathrm{mm}$ in length was inserted into the straight type crucible of 16 slits. A top part of the alloy was melted and confined by the exertion of the electromagnetic induction. Joule heats in the generator, coil, and the crucible were obtained from the increase in the temperature of cooling water and the flow rate.

\section{Results and Discussion}

\subsection{Electromagnetic Field in the Vertical Section}

4.1.1. Effect of Parameter $P_{12}$ on the Electromagnetic Field

Calculation of the electromagnetic field was performed for the straight type crucible with one slit under the coil current of $173 \mathrm{~A}$ and parameter $P_{i}=$ 0.97. Distributions of the effective magnetic flux density in the cases of parameter $P_{12}=0$ and $P_{12}=1$ are shown in Fig. 2. The magnetic flux density in the region between the coil and the external wall of the crucible and that in front of the centre of the slit in the crucible are relatively high. The maximum magnetic flux density in the crucible of parameter $P_{12}=1$ is 1.6 times as high as that of $P_{12}=0$. Almost the same difference in the effective magnetic flux density along the axis with the variation in parameter $P_{12}$ is observed, as shown in Fig. 3. Since, with the increase in the induced current the generation of the homogeneous scalar potential along the slit is assumed in the case of parameter $P_{12}=0$, the maximum magnetic flux density tends to decrease with the decrease of parameter $P_{12}$.

The magnetic flux density measured along the symmetry axis of the crucible with one slit was in agreement with that of the calculated results with parameter $P_{12}=1$. This means that the difference of

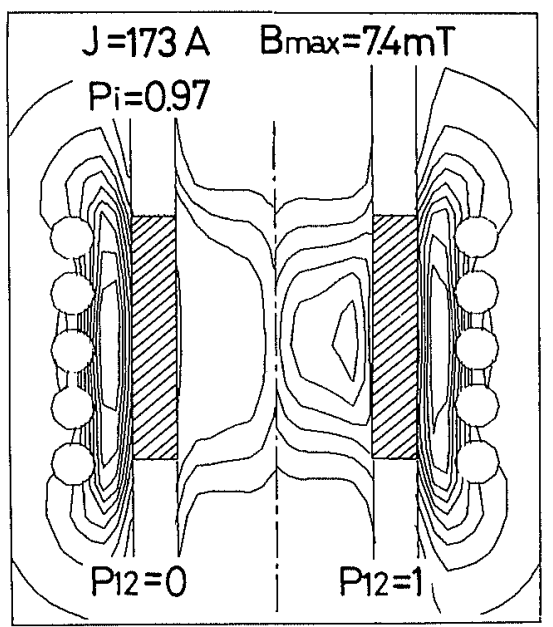

Fig. 2. Effect of parameter $P_{12}$ on the distribution of effective magnetic flux density in the vertical section of the crucible. 


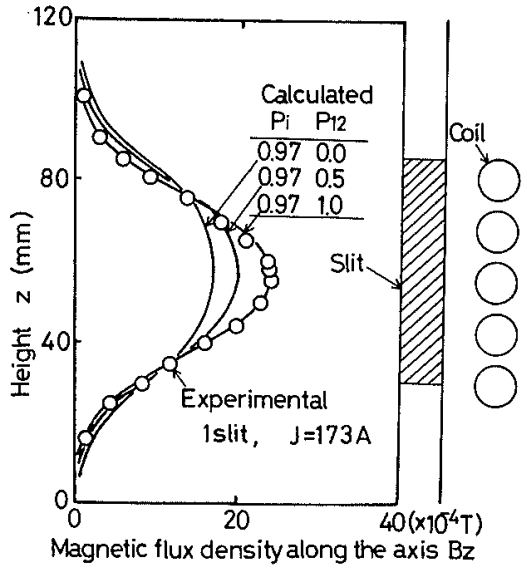

Fig. 3. Effect of parameter $P_{12}$ on the longitudinal distribution of magnetic flux density in the straight type crucible.

scalar potential between the adjacent slit is so small that it is hardly influential in the promotion of cross current in the slit.

Fig. 4 shows the effective magnetic flux density along the axis for the convergent type crucibles with one slit. From the calculation, the region where the magnetic flux density manifests the maximum value is expected to be shifted to the convergent part of the crucible diameter. Moreover, the shift length is significant, when $P_{12}=0$.

In the case of $P_{12}=1$, the shift of the region is considered to be the result of the concentration of the induced current into the space of small volume. In the case of $P_{12}=0$, this phenomenon is accompanied with the presence of the cross current, which leads to the increase in the shift length.

The experimental results for the magnetic flux density show the validity of the model with parameter $P_{12}=1$, which is the same as in the case of the straight type crucible. Hence, it is concluded that the significant cross current between the internal and the external sheets is not produced.

\subsubsection{Influence of Parameter $P_{i}$ on Electromagnetic Field}

Figs. 5 and 6 show the effective magnetic flux density along the axis for the slit numbers 1,8 , and 16 in the crucibles of straight and convergent types. The magnetic flux density increased with an increase in the number of slits, regardless of the difference of crucible shapes. It is found that the longitudinal variation of the magnetic flux density in the straight type crucibles can be described by the parameter $P_{i}=0.97,0.73$, and 0.48 under $P_{12}=1$ for the crucibles of 1,8 , and 16 slits, respectively. Moreover, it is found that the model with the same parameter enables to describe the magnetic flux density for the convergent type crucibles.

These results reveal the following.

(1) Regardless of the difference of crucible shapes and of the number of slits, the induced current on the internal and the external sheets of the crucible flows along the horizontal path.

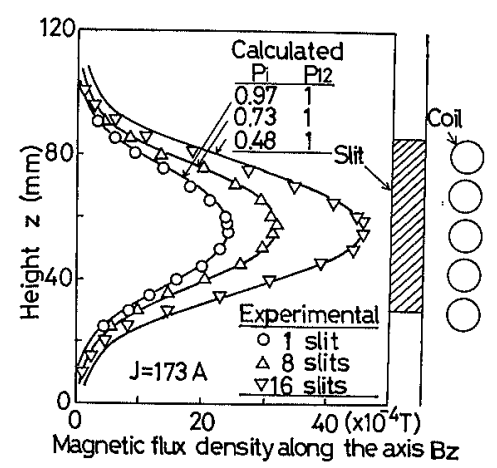

Fig. 5. Effect of parameter $P_{i}$ on the longitudinal distribution of magnetic flux density in the straight type crucible.
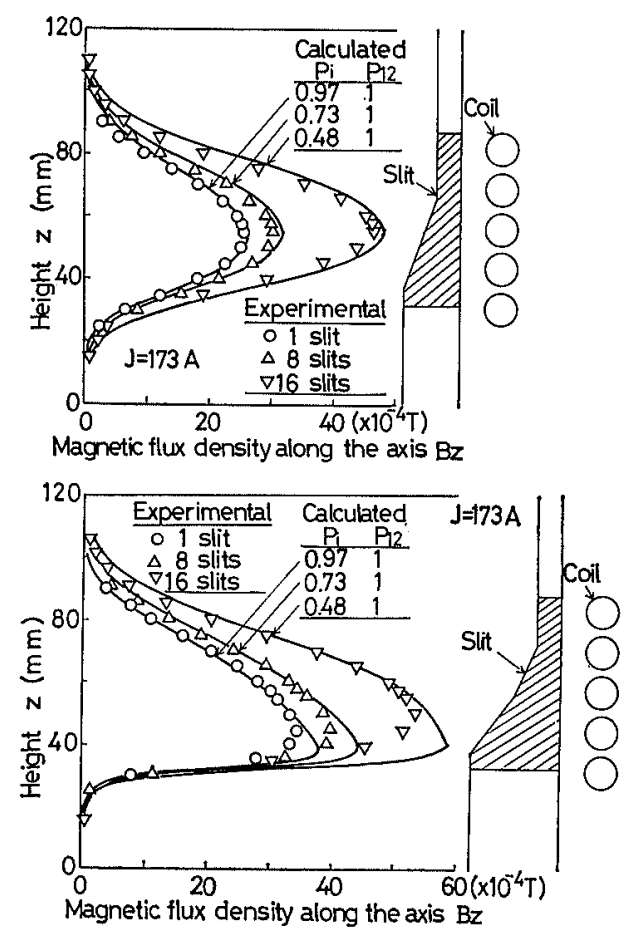

Fig. 6. Effect of parameter $P_{i}$ on the longitudinal distribution of magnetic flux density in the convergent type crucibles. 
(2) There is a possibility that the inductance between the crucible and the coil decreases with an increase in the number of slits, which leads to the increase in the current density.

\subsection{Magnetic Flux Density on the Horizontal Section}

As an example of the calculated results, the axial component of the effective magnetic flux density in the crucible with 16 slits is shown in Fig. 7. In this figure, the value along the specified direction in azimuth angle is also shown. The magnetic flux density in the crucible is found to be almost homogeneous. This agrees qualitatively as well as quantitatively with the solenoid theory that the magnetic flux density is homogeneous inside the solenoid and is zero outside. The magnetic flux density dramatically decreases inside the conductor because of the skin effect. Especially, the magnetic flux density between the coil and the crucible in the azimuthal direction where slits exist is high. This behaviour becomes significant with the decrease in the slit number.

Azimuthal distributions of the effective magnetic flux density at the internal radius of the crucibles with the slits of 2, 4, 8, and 16 are shown in Fig. 8. Since the magnetic flux enters into the crucible through the narrow slit between the segments, it is not homogeneous along the azimuthal direction but manifests itself higher especially on the direction where slit exits.

This means the possibility that the solidification in front of the segment would undergo at first followed by that in front of the slit where super heat and the pinch force are significant. Especially, when the heat loss through the segmented part of the crucible is significant, solidification proceeds from the bulk to the surface near the surface in front of the slit. This leads to the realization of casting which proceeds without infiltration of melt into the slit part of the crucible.

The magnetic flux density slightly increased with an increase in the slit number. This qualitatively agrees with the experimental results shown in Figs. 5

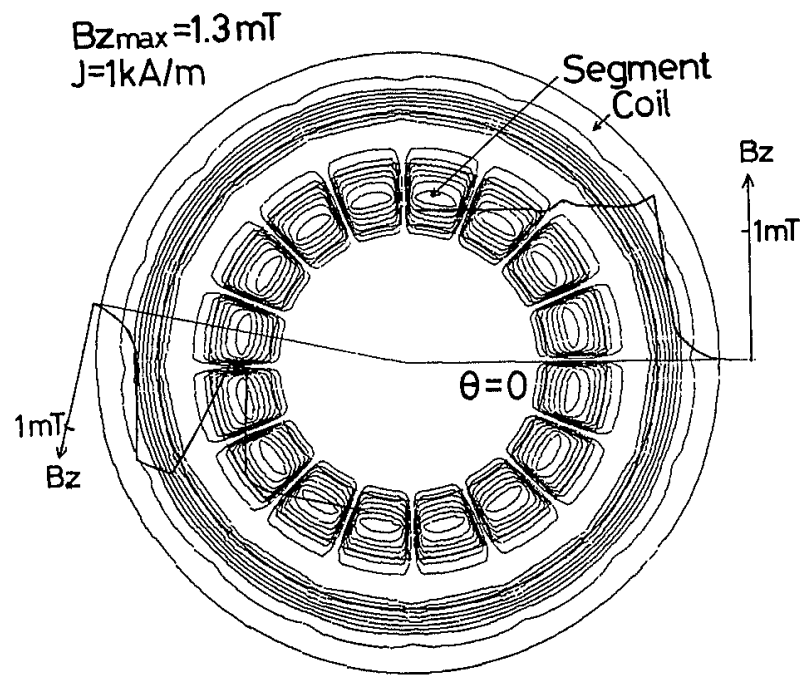

Fig. 7. Contour lines and radial distribution for the effective magnetic flux density in the horizontal section of the crucible. and 6 , but disagrees quantitatively. This means that the increase in the magnetic flux density with an increase in the slit number cannot be explained only by the decrease in the inductance mentioned in Sec. 4.1.2 and requires other reason. A remained possible reason is that the partition ratio of the induced current between the longitudinal region with and without the slit depends on such as the slit number, ratio of slit height to the coil height. The crucial mechanism must be await until a three dimensional model for the electromagnetic field is developed.

\subsection{Scalar Potential in the Horizontal Section}

As an example of the calculation, sum of the induced and the applied scalar potentials and partial distribution of the induced current at the instantaneous time are shown in Fig. 9. The scalar potential in the coil increases counterclockwise, $99.9 \%$ of which is the externally applied scalar potential. Most of the coil current flows clockwise in the inner side of the coil. The scalar potential in the segmented part of the crucible increases counterclockwise. Magnitude of the induced scalar potentials for the crucibles with 4 and 8 slits amounts to 8.5 and $2.6 \%$ of the scalar potential of the coil, respectively. In the external

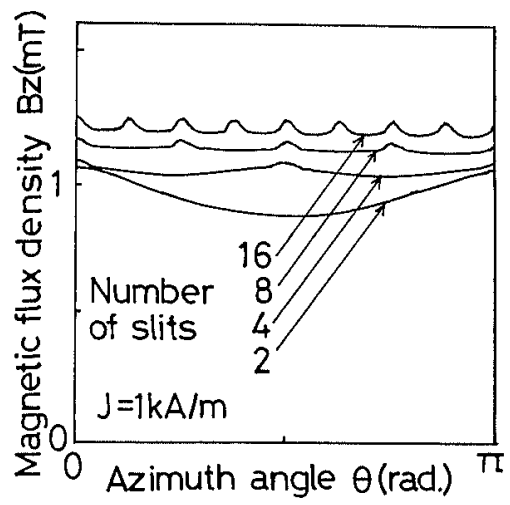

Fig. 8. Azimuthal distribution of effective magnetic flux density on the internal radius of the crucibles.

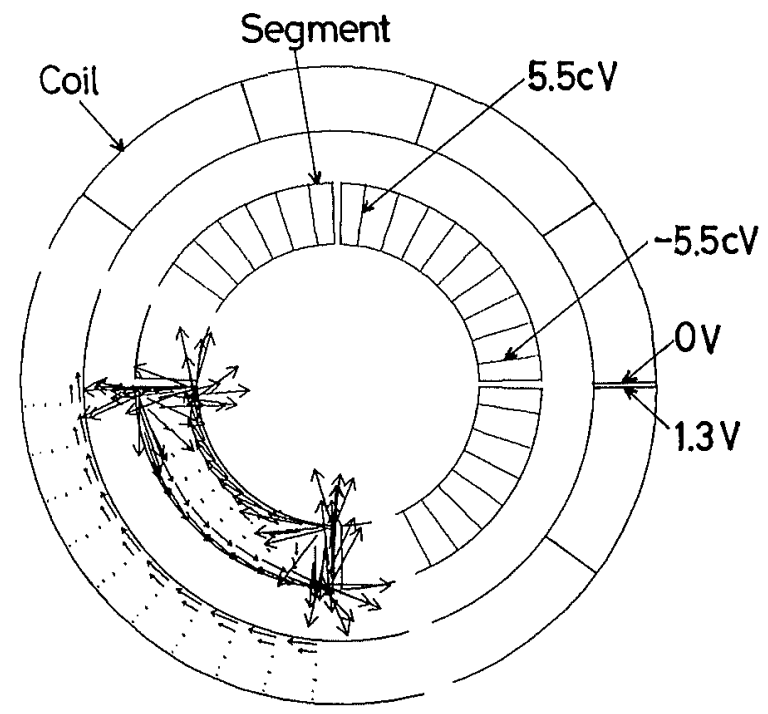

Fig. 9. Contour lines for the instantaneous scalar potential and current density. 
side of the segment, the induced current flows from the lower side of the scalar potential to the higher one. This is the reverse to the internal side of the segment.

It was mentioned in Sec. 4.1.1 that the model in the vertical section with parameter $P_{12}=1$ which corresponds to the absence of the scalar potential between adjacent slits successfully describes the experimental data. In order to explain this fact compatibly with the present result, it is necessary to consider that the inhomogeneous scalar potential is generated along the slit and it is large where the induced current density is high. Consequently, the significant reduction in the induced current does not take place by the existence of the scalar potential. This enables to describe the electromagnetic field by the model with apparently vanished scalar potential.

\subsection{Shape of the Melt}

Molten metal was relatively stable in the fusion of Al-Gu alloy. Experimentally obtained elevation height of the melt and the calculated shape of the melt together with the magnetic pressure exerted on the conductor surface under the melt volume of 44 and $65 \mu \mathrm{m}^{3}$, and the coil current of $1.4 \mathrm{kA}$ are shown in Fig. 10 .

The magnetic pressure is in action with the pinching manner of the conductor. Especially, it is significant on the surface of the coil placed in the middle and close to the external surface of the crucible. Along the crucible from top to bottom, the magnetic pressure gradually increases to its maximum value at the point where the surface of the melt and the internal wall of the crucible begin to touch, followed by the decrease. The calculated elevation height of the melt was in agreement with the experimental one. Since the melt is expected to contact with the internal wall near the slit bottom in the case of the straight type crucible, the solidification is preferable to be completed just above the bottom for the improvement of the ingot surface during continuous casting.

Vertical cross section of the Al-Cu alloy with the volume of $54 \mu \mathrm{m}^{3}$ above the slit bottom, which was obtained by rapid quench with Ar gas accompanied by the reduction of power of the generator, is shown in Fig. 11. It is found that the experimentally ob-

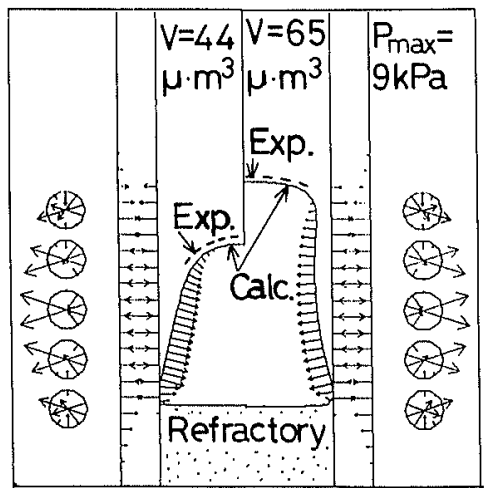

Fig. 10. Comparison of the calculated melt shape with the experimental one and the calculated magnetic pressure. tained shape is compatible with that lying between the volume of 44 and $65 \mu \mathrm{m}^{3}$ in the calculation.

Fig. 12 shows the distribution of the magnetic flux density in the presence of melt. Regions where the magnetic flux density is relatively high are observed between the crucible and the coil as well as the crucible and the melt. Especially, the latter region depends on the melt volume and the shape of the crucible, which is easily expected from the distribution of the magnetic flux density shown in Figs. 5 and 6.

\subsection{Energy Balance}

Energy balance in the system of the cold crucible and the coil voltage obtained from both the experiment and the calculation in the fusion of $\mathrm{Ti}-\mathrm{Al}$ alloy with the volume of $58 \mu \mathrm{m}^{3}$ above the slit bottom are shown in Table 2. The calculated results with respect to joule heat and the coil voltage agree with the experimental ones within $20 \%$ error. The joule heat and the coil voltage obtained from the calculation are small because of the absence of the induced current on the slit part of the crucible.

Since the ratio of the energy consumed in the melt to the accumulation of that in the coil, in the crucible and in the melt is $33 \%$, an increase of this value is desired.

\section{Conclusions}

Two kinds of advanced electromagnetic field

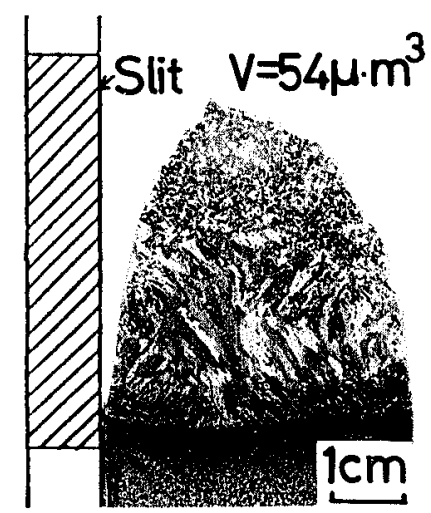

Fig. 11. Vertical section of Al-Cu alloy after cooled down with Ar gas.

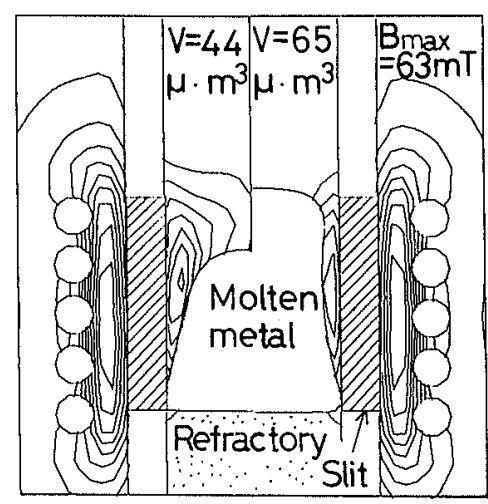

Fig. 12. Contour lines of the effective magnetic flux density in the vertical section. 
Table 2. Energy balance and induced voltage in the fusion of Ti-Al alloy.

\begin{tabular}{|c|c|c|c|}
\hline & & Experimental & Calculated \\
\hline Total power supply & $(\mathrm{kW})$ & 65 & - \\
\hline Generator loss & $(\mathrm{kW})$ & 33 & - \\
\hline Coil loss & $(\mathrm{kW})$ & 11.3 & 9.1 \\
\hline Grucible loss' 17 & $(\mathrm{~kW})$ & -- & 8.1 \\
\hline Liquid metal loss'2) & $(\mathrm{kW})$ & - & 8.4 \\
\hline 1) +2$)$ & $(\mathrm{kW})$ & 20.7 & 16.5 \\
\hline Generator voltage & $(\mathrm{V})$ & 230 & - \\
\hline Coil voltage & (V) & 136 & 110 \\
\hline
\end{tabular}

models for vertical and horizontal cross sections of the cold crucible have been developed. In the former model two independent parameters are introduced to describe the influence of the number of slits and the cross current between the internal and the external sheets. This model enables to obtain much information such as the shape of the melt, the electromagnetic field, and the coil voltage under the arbitrary axi-symmetric crucible shape. In the latter model, it is possible to describe the anisotropy of the magnetic field due to the presence of slits. The following conclusions were obtained through theoretical model calculation and experiments.

(1) Non-vanishing value of the scalar potential exists between adjacent slits of the crucible. However, it is hardly effective on the generation of cross current at the slit due to the inhomogeneous distribution of the scalar potential along the slit. This enables to develop the electromagnetic modelling on the simplified assumption of the horizontal path of the induced current on the crucible, regardless of the slit number and the shape of the crucible.

(2) An increase in the number of slits resulted in the increase of the magnetic flux density. Phenomenologically, this relation can be described by the use of the parameter which represents the intensity of the electromagnetic interaction and decreases with the increase in the slit number in the model of vertical section. However, the theoretical model for the horizontal section is not capable of quantitative description of the relation. The relation is mainly explained by the reason that the partition ratio of the induced current into the segmented part of the crucible increases with the number of slits.

(3) The magnetic flux density in the azimuthal direction of the slit at the internal radius of the crucible is expected to become higher. This means that there would be delay in solidification in front of the slit and the melt would not infiltrate into the slit.

(4) Since the melt is expected to contact with the internal wall near the slit bottom in the case of the straight type crucible, the solidification is preferable to be completed just above the slit bottom for the improvement of the ingot surface during continuous casting.

\section{Nomenclature}

A: vector potential $(T m=\mathrm{Vs} / \mathrm{m})$

B : magnetic flux density $\left(T=\mathrm{V}_{\mathrm{s}} / \mathrm{m}^{2}\right)$

$G$ : Green's function $(1 / \mathrm{m})$

$i$ : unit of an imaginary number $(-)$

$J:$ electric current (A)

$j:$ electric current density $\left(\mathrm{A} / \mathrm{m}^{2}\right)$

$P_{i}$ : parameter representing the magnitude of the electromagnetic interaction $(-)$

$P_{12}$ : parameter representing the degree of dependency of the current density in the internal sheet of the crucible on that in the external one (-)

$P_{M}:$ magnetic pressure $\left(\mathrm{Pa}=\mathrm{N} / \mathrm{m}^{2}\right)$

$P_{S}:$ surface tension $(\mathrm{Pa})$

$P_{H}:$ hydrostatic pressure of melt $(\mathrm{Pa})$

$P_{0}$ : uniform pressure on the melt $(\mathrm{Pa})$

$\mathbf{R}$ : coordinate of source point $(\mathrm{m})$

$\mathbf{r}$ : coordinate of observation point (m)

$V:$ volume $\left(\mathrm{m}^{3}\right)$

$\alpha$ : coefficient of surface tension $(\mathrm{N} / \mathrm{m})$

$\delta:$ skin depth (m)

$\varepsilon: \quad$ residual $(=\operatorname{div} \mathbf{j})\left(\mathrm{A} / \mathrm{m}^{3}\right)$

$\mu:$ magnetic permeability $(\mathrm{H} / \mathrm{m}=\mathrm{Vs} / \mathrm{Am})$

$\sigma:$ electric conductivity $(\mathrm{S} / \mathrm{m}=1 / \Omega \mathrm{m})$

$\phi: \quad$ scalar potential (V)

$\omega$ : angular frequency of an alternative current $(\mathrm{rad} / \mathrm{s})$

\section{REFERENCES}

1) Siemens and Halske A. G.: German Pat. 518499, Jan. 29, 1931.

2) P. Paillere, J-F. Wadier, Y. Boussant-Roux and M. Garnier: 6th World Conf. on Titanium, Société Française de Métallurgie, Les Ulis Cedex, France, (1988), 583.

3) D. Delage and R. Ernst: RGE, 4/83, (April, 1983), 266.

4) D. Delage, R. Ernst and J. Driole: IEEE-Trans, on Industrial Applications, (1981), 957.

5) A. Gagnoud and O. Sero Guillaume: E.D.F. Bull. de la Direction de Etudes et Recherches-Serie B Reseaux Electriques Materiels Electriques, (1986), No. 1, 41.

6) A. Gagnoud, J. Etay and M. Garnier: Trans. Iron Steel Inst. Jpn., 28 (1988), 36.

7) W.K.H. Panofsky and M. Phillips: Classical Electricity and Magnetism, Addison-Wesly P.C., Reading, MA, (1969), 243.

8) L. D. Landau and E. M. Lifšic: Elektrodinamika Splošnyh Sred, Gosudarstvennoc Izdatel'stvo Tehniko-teoreticeskoj Literatury, Moscow, (1957), 164.

9) Y. Sahai, J. E. Battles, R. S. Carbonara and C. E. Mobley: Casting of Near Net Shape Products, The Metall. Soc., Inc., (1988), 394-437.

10) B. D. Nichols and C. W. Hirt: J. Comput. P/yys., 8 (1971), 434.

11) G. W. Hirt, B. D. Nichols and N. C. Romero: Los Alamos Scientific Lab., Rep. No. 5852, (April, 1975).

(Originally published in Tetsu-lo-Hagané, 77 (1991), 345, in Japanese) 